\title{
Effects of impurities on the lattice dynamics of nanocrystalline silicon for thermoelectric application
}

\author{
Tania Claudio $\cdot$ Gabi Schierning $\cdot$ Ralf Theissmann • \\ Hartmut Wiggers $\cdot$ Helmut Schober . \\ Michael Marek Koza • Raphaël P. Hermann
}

Received: 28 May 2012/Accepted: 21 August 2012/Published online: 19 September 2012

(C) The Author(s) 2012. This article is published with open access at Springerlink.com

\begin{abstract}
Doped silicon nanoparticles were exposed to air and sintered to form nanocrystalline silicon. The composition, microstructure, and structural defects were investigated with TEM, XRD, and PDF and the lattice dynamics was evaluated with measurements of the heat capacity, of the elastic constants with resonant ultrasound spectroscopy and of the density of phonon states (DPS) with inelastic neutron scattering. The results were combined and reveal that the samples contain a large amount of silicon dioxide and exhibit properties that deviate from bulk silicon. Both in the reduced DPS and in the heat capacity a Boson peak at low energies, characteristic of amorphous $\mathrm{SiO}_{2}$, is observed. The thermal conductivity is strongly reduced due to nanostructuration and the incorporation of impurities.
\end{abstract}

T. Claudio - R. P. Hermann

Jülich Centre for Neutron Science, JCNS and Peter Grünberg Institut PGI, JARA-FIT, Forschungszentrum Jülich GmbH, Jülich 52425, Germany

R. P. Hermann

e-mail: r.hermann@fz-juelich.de

T. Claudio $(\bowtie) \cdot$ R. P. Hermann

Faculté des Sciences, Université de Liège, Liège 4000, Belgium

e-mail: t.claudio.weber@fz-juelich.de

G. Schierning $\cdot$ R. Theissmann $\cdot$ H. Wiggers

Faculty of Engineering and Center for NanoIntegration

Duisburg-Essen (CeNIDE), University of Duisburg-Essen,

47057 Duisburg, Germany

H. Schober - M. M. Koza

Institut Laue Langevin, 6 rue Jules Horowitz, BP 156,

38042 Grenoble, France

\section{Introduction}

With increasing availability of tailored nanopowders and nanostructures, densified assemblies of engineered nanostructures are focussed upon for many possible applications, as for instance thermoelectricity. Thermoelectric devices provide cooling when an applied current pumps heat from the cold side towards the hot side through the Peltier effect, or enable waste heat recovery by converting a heat gradient to electrical power through the Seebeck effect. A good thermoelectric material should possess a large Seebeck coefficient, $S$, a high electrical conductivity $\sigma$, and low thermal conductivity $\kappa$ to maximize the dimensionless figure of merit $\mathrm{ZT}=S^{2} \sigma T / \kappa$ for the thermoelectric performance of a material [1-3]. Reducing the thermal conductivity is therefore a natural way to improve the performance of a thermoelectric material.

Silicon-based materials combine several desirable properties for thermoelectric applications: most are nontoxic in contrast to many other thermoelectric materials and the raw material is relatively inexpensive and available in industrial quantities. Alloys of $\mathrm{Si}-\mathrm{Ge}$ have a competitive figure of merit at high temperatures [1, 2, 4]; and silicon nanowires with a rough surface present a drastic reduction in thermal conductivity resulting in a $\mathrm{ZT} \approx 0.6$ to 1 at room temperature $[5,6]$. Furthermore, the properties and technology around silicon are well established in the semiconductor industry, making it an ideal thermoelectric model material for which reference data from single crystals can be used as comparison.

Due to its exceptionally large thermal conductivity, $156 \mathrm{~W} / \mathrm{m} \mathrm{K} \mathrm{[7]} \mathrm{at} \mathrm{room} \mathrm{temperature,} \mathrm{predominantly} \mathrm{lattice}$ thermal conductivity, silicon is the perfect model material to test the improvement of thermoelectric properties through nanostructuration, when the thermal conductivity 
is decreased due to the creation of scattering centers for phonons. This concept was already successfully applied to a number of thermoelectric materials $[3,4,8,9]$. Nanocrystalline silicon was demonstrated to be a competitive thermoelectric material with best $\mathrm{ZT}=0.7$ at $1275 \mathrm{~K}$ [8]. Furthermore, the addition of impurities and/or defects was shown to result in a favorable decrease of the lattice thermal conductivity of silicon. Gibbons et al. [10] used first-principle methods to calculate the thermal conductivity of nanostructured $\mathrm{Si}$ which contained defects and/or impurities and concluded that there exist narrow ranges of impurity parameters (mass, bond strength, etc.) for which a reduction in the thermal conductivity was predicted. Such a decrease was previously observed [11] on bulk silicon as a function of carrier concentration. A $5 \%$ substitution of $\mathrm{Ge}$ on nanostructured $\mathrm{Si}$ proved to efficiently scatter phonons with a wavelength shorter than $1 \mathrm{~nm}$ [4], resulting in a further reduction of the thermal conductivity.

Previous study by Schierning et al. [12] demonstrated that nanoparticles of Si produced by a gas-phase synthesis and exposed to air before sintering present a certain amount of impurities in the form of amorphous $\mathrm{SiO}_{2}$.

Here, we report on the role of such impurities on the lattice dynamics of nanocrystalline Si produced by a bottom-up process with respect to thermoelectric applications.

\section{Experimental}

Silicon nanoparticles were synthesized by a plasma-assisted gas-phase process, using a microwave reactor [13]. By variation of microwave power, chamber pressure, and concentration of the precursor gas silane $\left(\mathrm{SiH}_{4}\right)$ as well as the plasma gases $\mathrm{Ar}$ and $\mathrm{H}_{2}$, almost spherically shaped particles were obtained. The conditions used were a precursor concentration of 100-200 vpm, pressures of 20-100 mbar, and microwave powers of 1200-2000 W. Two different batches of crystalline particles of pre-defined dimension (22 and $30 \mathrm{~nm}$ ) in a narrow size distribution were obtained by controlling these parameters. N-type doping was achieved by adding phosphine $\left(\mathrm{PH}_{3}\right)$ to the precursor gas. The incorporation of electrically active phosphorus into the nanoparticles was demonstrated for similar batches of silicon nanoparticles by electron paramagnetic resonance (EPR) earlier [14].

The compaction of the nanoparticles was done with a spark plasma sintering furnace from FCT Systeme GmbH. Several grams of the nanoparticles of the two different batches were pre-compacted and subsequently sintered to dense pellets with a diameter of $2 \mathrm{~cm}$ in a mbar $\mathrm{Ar}$ atmosphere. Heating and cooling rates were fixed to $100 \mathrm{~K} / \mathrm{min}$. The sintering temperature was $1050{ }^{\circ} \mathrm{C}$ with a hold time of $3 \mathrm{~min}$. A pressure of $35 \mathrm{MPa}$ was applied during sintering. No special care was taken to prevent samples from contamination with oxygen.

Structural characterization of raw powder and sintered pellets was done by transmission electron microscopy (TEM) with a $200 \mathrm{keV}$ Philips, Tecnai F20 ST microscope. Sintered pellets were cut into pieces, mechanically thinned, and subsequently polished by a precision ion polishing system with Ar-ions.

The average crystallite size of the nanoparticles and the nanocrystalline pellets was obtained by X-ray diffraction (XRD) and a subsequent Rietveld refinement based on the measured data and taking into consideration the Debye Scherrer broadening of the diffraction peaks. For the XRD, pieces of the sintered pellets were thoroughly ground to ensure good powder statistics and the powder was filled into glass capillaries. A Siemens D5000 powder diffraction machine was used in transmission geometry, equipped with a Germanium monochromator and a PSD-50 M detector (MBraun). The refinement of the structural parameters was done using the program FULLPROF [15]. Rietveld refinement was done using five parameters: zero-shift, lattice parameter, and three parameters for the peak shape (the Lorentzian profile parameters $X$ and $Y$, and Gaussian profile parameter $G$ ).

As the contribution from an amorphous phase can not be determined directly from XRD, data for an analysis of the pair distribution function (PDF) were obtained with synchrotron radiation in transmission through a $1 \mathrm{~mm}$ sample at the high energy station 6-ID-D of the advanced photon source (APS) at Argonne National Laboratory. The X-ray wavelength was $0.142013 \AA$ and the $2 \mathrm{D}$ circular image plate (IP) camera Mar345 was positioned at a distance of $218.7 \mathrm{~mm}$ from the sample, as determined by a NIST SRM640c Si standard. No preferential orientation was observed. The data were reduced to diffraction patterns with the program FIT2D [16], corrected and processed to get the PDF with the PDFgetX2 program package [17] and refined over a range from 1.1 to $30 \AA$ with the program PDFgui [18].

Inelastic neutron scattering experiments were performed on both samples on the time-of-flight (TOF) spectrometer IN6 at the cold source of the high-flux reactor at the Institute Laue-Langevin (Grenoble, France). The incident wavelength was $5.12 \AA$ with an energy resolution of 0.13 $\mathrm{meV}$, determined by the elastic neutron scattering on a vanadium sample. Although the resolution function broadens rapidly with increasing neutron energy, good and reliable data was obtained up to $140 \mathrm{meV}$. The IN6 spectrometer supplies a typical flux of $10^{6} \mathrm{n} / \mathrm{cm}^{2} \mathrm{~s}$ on the sample, with a beam size of $25 \times 45 \mathrm{~mm}^{2}$.

The same measurements were additionally performed on a crushed monocrystalline silicon wafer which was also 
phosphorus doped, with a nominal resistivity of $0.3 \Omega \mathrm{cm}$, for comparison with a polycrystalline sample.

Due to the presence of impurities in the samples, only an estimation of the density of phonons states (DPS) was extracted from the scattering function $S(\omega)$ in the inelastic scattering approximation and corrected for multiphonon scattering using the program LAMP [19]. The DPS of the polycrystalline $\mathrm{Si}$ was normalized to 1 between 0 and $73 \mathrm{meV}$ and the DPS of the nanocrystalline Si samples were rescaled according to the peaks at 20 and $60 \mathrm{meV}$, as additional contributions from hydrogen and oxides impede other means of normalization.

Measurements of the heat capacity were done on both sintered pellets between 0.1 and $400 \mathrm{~K}$ in a commercial physical property measurement system (PPMS) from Quantum Design, using the dilution option for measurements between 0.1 and $2 \mathrm{~K}$.

The elastic constants were obtained through resonant ultrasound spectroscopy (RUS), a non-destructive technique which records the frequencies of the natural modes of vibration by using two piezoelectric transducers: one which excites the sample and the other which detects the resonant response of the sample [20]. The $C_{11}$ and $C_{44}$ components of the elastic tensor were calculated from the first 30 resonant frequencies and fully characterize the elasticity in such an elastically isotropic sample. An in-house equipment was used with a parallelepiped-shaped sample geometry of $\sim 2.0 \times 1.5 \times 1.5 \mathrm{~mm}^{3}$. All measurements were performed at room temperature.

Macroscopic measurements of the resistivity, Seebeck coefficient, and thermal conductivity were performed on both pellets between 2 and $300 \mathrm{~K}$ using the thermal transport option of the QD-PPMS.

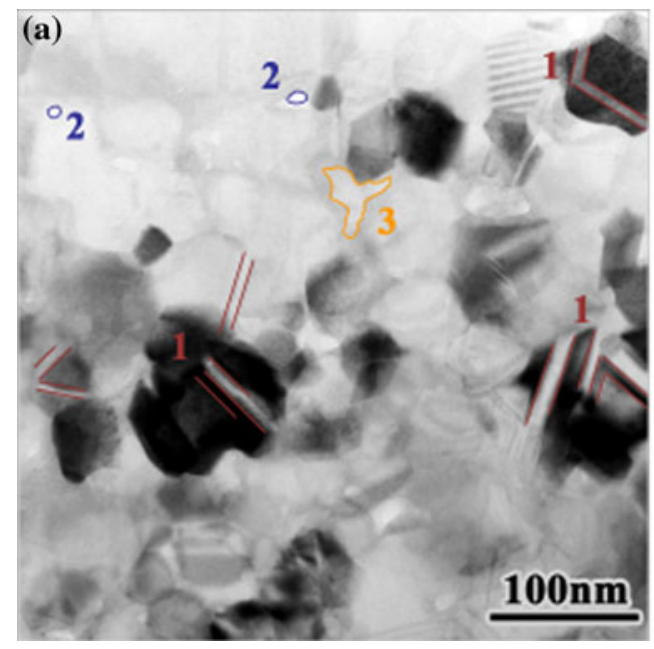

Fig. 1 a TEM bright field image of a silicon nanocomposite with mean crystallite size of $40 \mathrm{~nm}$. Characteristic microstructural features are twins (marked with 1), pores (marked with 2), and oxidic

\section{Results and discussion}

Microstructure of the nanocrystalline silicon

A typical TEM bright field image of the silicon nanocomposite is shown in Fig. 1a. Characteristic features of the microstructure are nanocrystalline grains. Planar defects as seen in Fig. 1a, marked with 1, originate from the gas phase synthesis: the silane precursor decomposes, which results in nucleation, coalescence, and growth of nanoparticles. A fingerprint of the coalescence is a large twinning of the initial nanopowder and, as a consequence, of the nanocomposite. A porosity of $\sim 2-3 \%$ is typical for silicon nanocomposites from this process [21]. A thin native oxide shell is the consequence of handling the silicon nanopowder under ambient conditions. This native oxide shell rearranges during the densification and forms oxidic precipitates within a three-dimensional interconnecting silicon network [12]. In Fig. 1a, two pores and one oxidic precipitate are exemplary marked with 2 and 3 , respectively. A high resolution image of a triple point between neighboring silicon grains shows that the siliconsilicon interfaces are free of oxygen (Fig. 1b).

Information about the average crystallite size of the initial nanopowder batches and of the compacted nanocomposites was obtained from XRD and Rietveld refinement and it was found to be 22 and $30 \mathrm{~nm}$ for the nanopowder and 30 and $40 \mathrm{~nm}$, respectively (Fig. 2), for the pellets, due to coarsening during the sintering process. For very similar samples (i.e., produced with comparable parameters of powder synthesis and sintering), a quantitative TEM analysis was done and compared with the microstructural model obtained by XRD [21]. This study

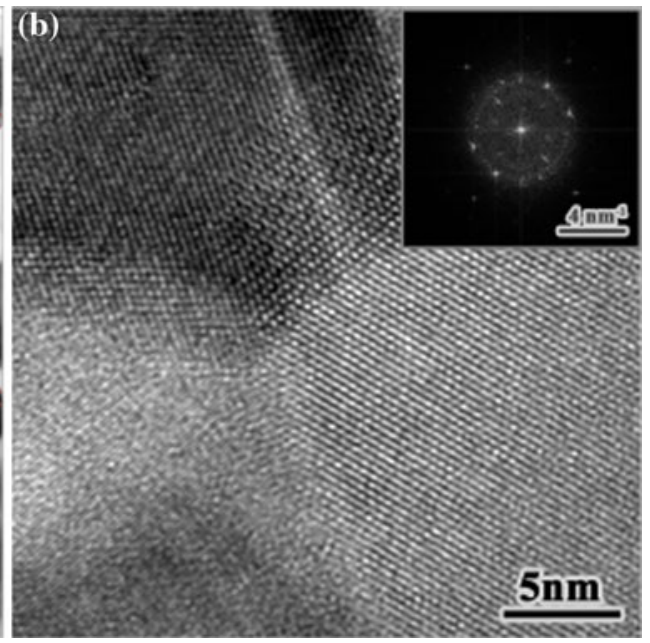

precipitates (marked with 3). b High resolution image of a triple point between silicon grains. Inset The Fourier transform of $\mathbf{b}$ 


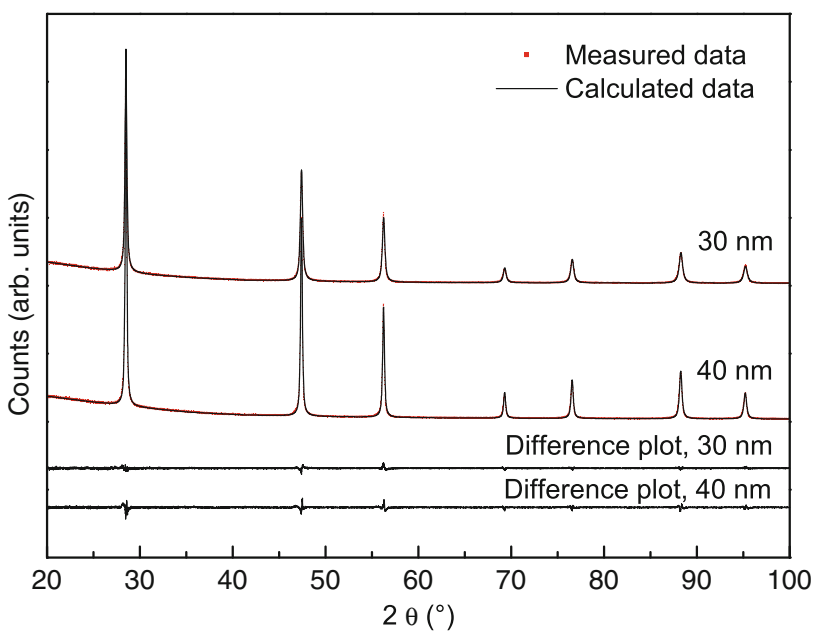

Fig. 2 XRD patterns of the sintered pellets, together with the calculated structure model obtained from a Rietveld refinement and difference plot between measured and calculated data. A good agreement between refined structure model and measured data can be seen. The microstructural model results in 30 and $40 \mathrm{~nm}$ average crystallite size of the two samples

revealed a good agreement between average crystallite size obtained from XRD and from quantitative TEM analysis, but showed that the size distribution of the crystallites broadens with mean crystallite size.

PDF analysis reveals the presence of amorphous $\mathrm{SiO}_{2}$ in both nanocrystalline samples (Fig. 3). The first near neighbor distance of $\mathrm{Si}-\mathrm{Si}$ is $2.35 \AA$, but an additional peak at $1.60 \AA$ corresponds to the $\mathrm{Si}-\mathrm{O}$ distance of crystalline alpha quartz. The data was modelled with a crystalline $\mathrm{Si}$ $(F d 3 m)$ phase and, as amorphous $\mathrm{SiO}_{2}$ is expected to have the same short-range order as crystalline $\mathrm{SiO}_{2}$, a crystalline alpha quartz $\left(P 3_{1} 21\right)$ phase with a strongly reduced particle size, refined to $\approx 9 \AA$, to account only for the first peaks of quartz. Four peaks belonging to the amorphous $\mathrm{SiO}_{2}$ are

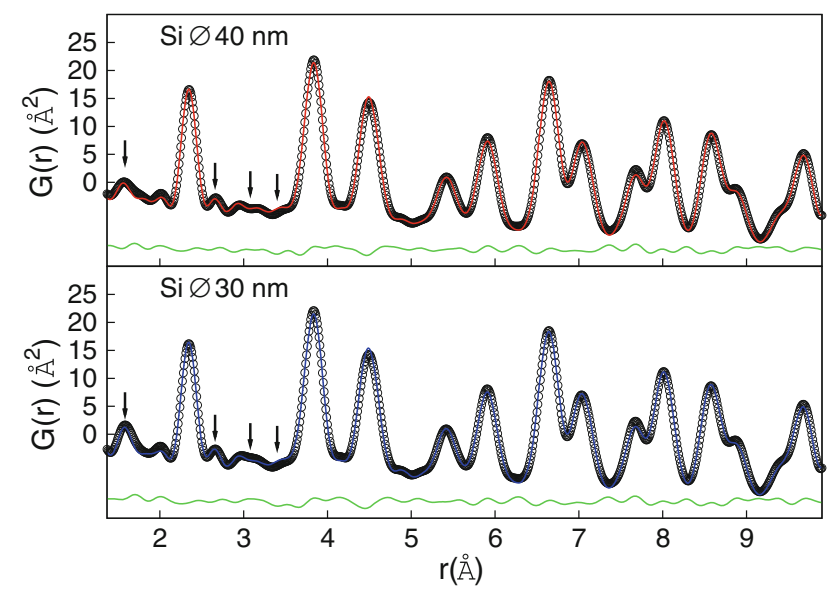

Fig. 3 PDF for both nanocrystalline Si samples. Arrows indicate the first four $\mathrm{SiO}_{2}$ peaks, points are the data obtained while red and blue are the fit. The green line is the difference between data and fit (Color figure online) clearly visible below $3.5 \AA$ and are indicated by arrows in Fig. 3. Refinement of the PDF data gave a quantity of 15(1) $\%$ of $\mathrm{SiO}_{2}$ for the nanocrystalline sample with $30 \mathrm{~nm}$ and $9.6(5) \%$ for the nanocrystalline with $40 \mathrm{~nm}$. The different amounts of oxide within both samples can be attributed to a higher surface-to-volume ratio of the raw powder with smaller initial particle size. Note that a $9.6(5) \%$ content of oxide in the $40 \mathrm{~nm}$ sample is in excellent agreement with a TEM tomography study with three-dimensional data reconstruction in which a very comparable sample had an oxide content of $9 \%$ [12].

\section{Estimation for the DPS}

An estimation for the DPS of the two nanocrystalline silicon samples obtained from inelastic neutron scattering, shown in Fig. 4, deviates significantly from the DPS of the reference polycrystalline silicon sample which presents a smooth $E^{2}$ dependence at the low energy region, better observed on the reduced DPS (inset of Fig. 4) and has an cutoff energy of $73.4 \mathrm{meV}$. In contrast, the DPS of the nanocrystalline $\mathrm{Si}$ samples present a significant contribution at low energies corresponding to a pronounced peak between 2.5 and $13 \mathrm{meV}$ in the reduced DPS, $g(E) / E^{2}$, as well as additional peaks at around 30,50 , and $80 \mathrm{meV}$ on the DPS $(g(E))$, and a broad feature at around $110 \mathrm{meV}$.

We interpret the specific features of the DPS as follows:

A pronounced peak observed between 2.5 and $13 \mathrm{meV}$ in the reduced DPS, see inset of Fig. 4, is attributed to an amorphous phase, and corresponds to the Boson peak usually observed in glasses [22]. This peak originates from the amorphous $\mathrm{SiO}_{2}$ present in the sample and is in good agreement with the Boson peak position previously observed on amorphous $\mathrm{SiO}_{2}$ with Raman scattering

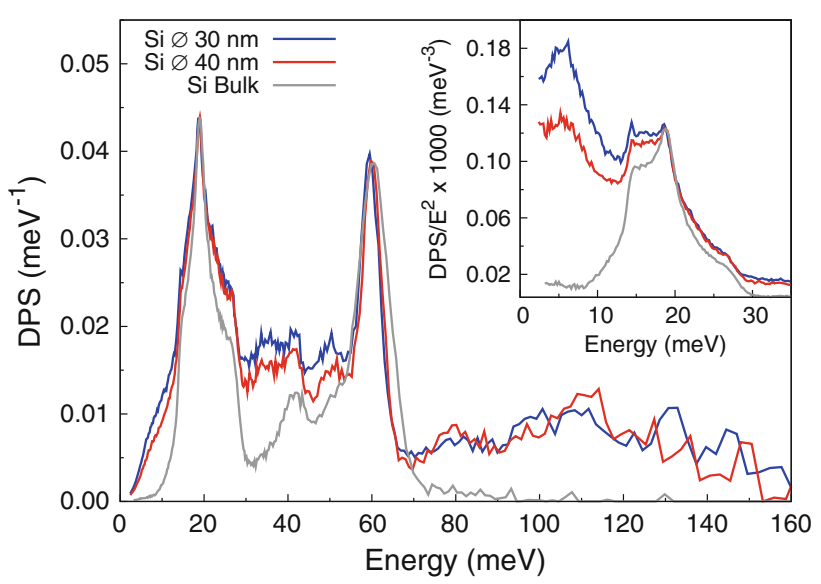

Fig. 4 DPS for both nanocrystalline Si compared with reference polycrystalline Si. Inset The reduced DPS, where the Boson peak around $6 \mathrm{meV}$ is a clear indication of amorphous $\mathrm{SiO}_{2}$ 
$\left(51 \mathrm{~cm}^{-1}=6.3 \mathrm{meV}\right)$ [23] and with neutron scattering measurements (between 2 and $6 \mathrm{meV}$ ) [24].

The peak at around $30 \mathrm{meV}$ is also associated to amorphous $\mathrm{SiO}_{2}$, as previously observed by Fabiani et al. [24].

The mode with highest energy in the DPS of bulk Si is $\approx 60 \mathrm{meV}$. The measured values of the DPS at higher energies and especially a broad feature at around $110 \mathrm{meV}$ strongly suggest the presence of hydrogen in the nanocrystalline sample [25, 26]. A peak at $80 \mathrm{meV}$ corresponds to the rocking mode of $\mathrm{H}$ atoms compensating a dangling Si-bond, while a prominent peak at $110 \mathrm{meV}$ belongs to a bending mode of $\mathrm{SiH}_{2}$ in which the bond angle $\mathrm{H}-\mathrm{Si}-\mathrm{H}$ is modulated $[25,26]$. The $\mathrm{Si}-\mathrm{H}$ modes observed in this experiment are in good agreement with previous measurements done with inelastic neutron scattering [25] and IR absorption spectrum on sputtered hydrogenated amorphous Si [26].

When the nanopowder is exposed to air, not only a shell of $\mathrm{SiO}_{2}$ is formed around the nanoparticles due to silicon's strong affinity for oxygen but also a significant amount of water is adsorbed which could lead to the thermal oxidation of silicon with $\mathrm{H}_{2}$ and $\mathrm{SiO}_{2}$ as reaction product:

$\mathrm{Si}+2 \mathrm{H}_{2} \mathrm{O} \longrightarrow \mathrm{SiO}_{2}+2 \mathrm{H}_{2(\mathrm{~g})}$

which may explain the large amount of $\mathrm{SiO}_{2}$ within the investigated samples. Furthermore, under the sintering conditions, the surplus molecular hydrogen may dissociate into $\mathrm{H}$ atoms being incorporated at interstitial sites of $\mathrm{Si}$ [27].

To conclude, the specific features found in the DPS of the investigated nanocrystalline silicon are due to contributions of $\mathrm{SiO}_{2}$ impurities at lower energies, whereas contributions above $75 \mathrm{meV}$ are attributed to $\mathrm{H}$ impurities.

As an additional analysis, the sound velocity was extracted from the DPS using the low energy limit of $g(E) / E^{2}[28]:$

$v_{\mathrm{s}}^{3}=\frac{E^{2}}{2 \pi^{2} N_{\mathrm{V}} \hbar^{3} g(E)}$,

where $N_{\mathrm{V}}$ is the number of atoms per unit volume $\left(N_{\mathrm{V}}=\right.$ $\rho N_{\mathrm{a}} / M_{\mathrm{w}}$ with $\rho$ being the density, $N_{\mathrm{a}}$ the Avogadro number and $M_{\mathrm{w}}$ the molecular weight).

Due to the presence of the Boson peak on the nanocrystalline Si samples, only a higher limit of $g(E) / E^{2}$ for $E \rightarrow 0$, i.e., lower limit of the sound velocity, can be calculated. The results are listed in Table 2 . The value obtained for bulk Si by this method $(6.73 \mathrm{~km} / \mathrm{s})$ is in good agreement with the value calculated from the elastic constants [29] $(5.94 \mathrm{~km} / \mathrm{s})$.

Influence of oxide impurities on heat capacity

The heat capacity of the nanocrystalline silicon samples was measured between 0.1 and $400 \mathrm{~K}$ and the data is shown in the $C_{\mathrm{p}} / T^{3}$ versus $T$ representation in Fig. 5.

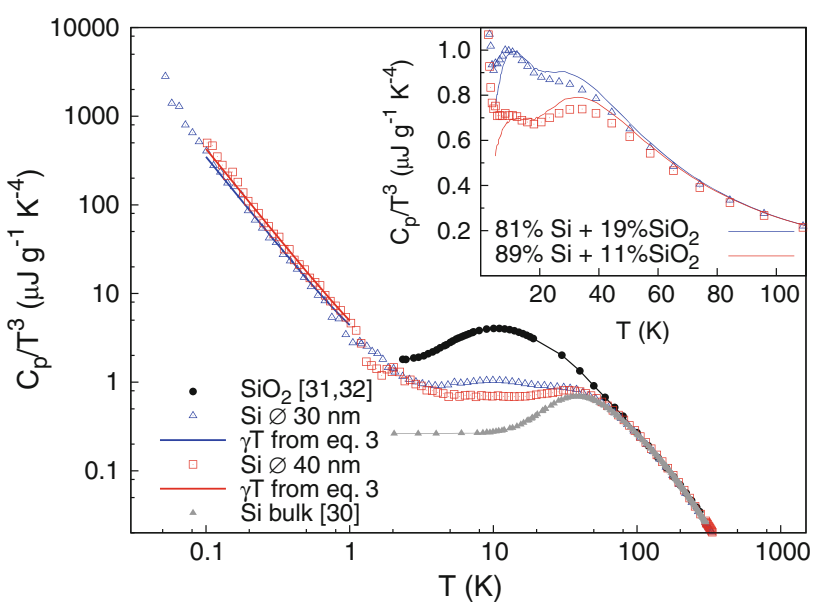

Fig. 5 Heat capacity divided by $T^{3}$ for both nanocrystalline $\mathrm{Si}$ compared with crystalline $\mathrm{Si}[30]$ and amorphous $\mathrm{SiO}_{2}[31,32]$ in double logarithmic representation. Inset Interpolated fit considering the indicated mixture of $\mathrm{Si}$ and $\mathrm{SiO}_{2}$ (error bars are smaller than the symbol size)

The Debye $T^{3}$ approximation valid at low temperatures for lattice specific heat is given by

$C_{\mathrm{V}}=\frac{12 \pi^{4}}{5} N k_{\mathrm{B}}\left(\frac{T}{\Theta_{D}}\right)^{3}$

Experimentally, the heat capacity is obtained at constant pressure $\left(C_{\mathrm{p}}\right)$. For a solid $C_{\mathrm{p}}$ and $C_{\mathrm{V}}$ differ by significantly less than a percent at low temperatures [33].

For bulk crystalline $\mathrm{Si}$ [30] $C_{\mathrm{p}} / T^{3}$ reaches a constant value for $T \rightarrow 0$, whereas both nanocrystalline $\mathrm{Si}$ samples show a pronounced deviation from the Debye $T^{3}$ law.

In contrast, between 5 and $15 \mathrm{~K}$, a characteristic maximum substitutes the plateau observed for the bulk $\mathrm{Si}$ sample in the case of the two nanocrystalline samples. The maxima can be attributed to a Boson peak characteristic for amorphous materials [34], which is more pronounced for the sample with smaller nanocrystallites. This feature can be related to the presence of amorphous $\mathrm{SiO}_{2}$ [31] within the samples, in line with the previous statement about the larger content of $\mathrm{SiO}_{2}$ in the sample which was produced from smaller nanoparticles.

In addition, a steep increase in $C_{\mathrm{p}} / T^{3}$ for $T \rightarrow 0$ is observed for both nanocrystalline samples, which indicates that at very low temperatures, below $2 \mathrm{~K}$, an additional contribution proportional to $T^{\alpha}, \alpha<3$, is present. At a carrier concentration of $10^{20} \mathrm{~cm}^{-3}$, there is no freeze-out of the carriers, and an electronic contribution is expected at low temperatures. Such contribution can be estimated with:

$C_{\mathrm{p}}=\gamma T+\beta T^{3}$,

where $\gamma$ accounts for the electronic contribution and $\beta$ for the lattice contribution. 
A fit with the above equation for the data below $T=15 \mathrm{~K}$ yielded values of $\gamma=0.097(2) \mathrm{mJ} \mathrm{mol}^{-1} \mathrm{~K}^{-2}$ for the sample with smaller nanocrystallites and $\gamma=0.120(2) \mathrm{mJ}$ $\mathrm{mol}^{-1} \mathrm{~K}^{-2}$ for the sample with larger nanocrystallites. These values are only one order of magnitude smaller than the values calculated and obtained for metals (0.6-2.5 mJ mol $\left.\mathrm{mJ}^{-1} \mathrm{~K}^{-2}[35]\right)$.

Two approaches were used to model the $T^{3}$ contribution quantitatively. First, a sum of the interpolated data of the bulk $\mathrm{Si}$ [30] and $\mathrm{SiO}_{2}$ [31, 32] heat capacities using the weight percentages as free fitting parameter (inset of Fig. 5) which resulted in a value of 11 and $19 \%$ of $\mathrm{SiO}_{2}$ for the samples with smaller and larger nanocrystallite sizes, respectively, differing only slightly from the value extracted from the PDF refinement (9.6 and $15 \%$ ). This deviation occurred because two contributions to the heat capacities at low temperatures were not considered in this fit: the electronic contribution due to a high concentration of dopants, and nanostructuration as previously observed for $\mathrm{SiO}_{2}$ [36].

A second approach was to model the data above $2 \mathrm{~K}$. While the Debye model for the heat capacity can correctly describe the high temperature $\left(C_{\mathrm{p}}\right)$, a second term has to be added to describe the excess $C_{\mathrm{p}}$ at $\sim 45 \mathrm{~K}$ caused by a flat acoustic mode in the dispersion curve, characteristic for any form of silicon [37, 38]. This term is better modelled with the Einstein model $\left(C_{\mathrm{E}}\right)$. A third term was necessary to account for the Boson peak caused by the amorphous $\mathrm{SiO}_{2}$ contribution and can also be modelled with an Einstein term $\left(C_{\mathrm{BP}}\right)$ :

$$
\begin{aligned}
& C_{\mathrm{p}}(T)=\mathrm{d} C_{\mathrm{D}}(T)+e C_{\mathrm{E}}(T)+b C_{\mathrm{BP}}(T) \\
& C_{\mathrm{D}}(T)=9 N k_{\mathrm{B}}\left(T / \Theta_{\mathrm{D}}\right)^{3} \int_{0}^{\Theta_{\mathrm{D}} / T} \frac{e^{x} x^{4}}{\left(e^{x}-1\right)^{2}} \mathrm{~d} x \\
& C_{\mathrm{E}}(T)=3 N k_{\mathrm{B}} \frac{e^{\Theta_{\mathrm{E}} / T}\left(\frac{\Theta_{\mathrm{E}}}{T}\right)^{2}}{\left(e^{\Theta_{\mathrm{E}} / T}-1\right)^{2}},
\end{aligned}
$$

where $N$ is the number of atoms in the solid, $k_{\mathrm{B}}$ is Boltzmann's constant, $d, e$, and $b$ are the pre-factors for the Debye, Einstein, and Boson peak contributions, respectively, and $\Theta_{\mathrm{D}}, \Theta_{\mathrm{E}}$, and $\Theta_{\mathrm{BP}}$ are the Debye, Einstein, and Boson peak temperatures, respectively. More details about the Debye and Einstein models can be found in [39].
The literature value for the bulk Debye temperature $(625 \mathrm{~K})$ was determined by fitting the observed $C_{\mathrm{p}}$ to the Debye formula at the point where $C_{\mathrm{p}}=3 n k_{\mathrm{B}} / 2$ [33] and is the same as the one obtained from the Debye plateau at low temperatures. A fit with an Einstein and a Debye term for the entire range of temperatures gave a Debye temperature of 745(2) K and an Einstein temperature of 202(3) $\mathrm{K}$ resulting in an Einstein energy of $17.4(3) \mathrm{meV}$, which is in very good agreement with the same feature observed in this energy in the reduced DPS.

Further fittings of the data from the nanocrystalline samples showed values for the Einstein, Debye and Boson peak temperatures as summarized in Table 1 . The values obtained for the Einstein temperatures were also converted to energy $(1 \mathrm{~K}=0.0862 \mathrm{meV})$. Note that the Boson peak energies are in very good agreement with the features at smallest energy in the reduced DPS, see inset of Fig. 4.

A calculation of the sound velocity from the Debye temperature can be done using the equation [35]:

$v_{\mathrm{s}}=\frac{k_{\mathrm{B}} \Theta_{\mathrm{D}}}{\hbar\left(6 \pi^{2} N_{\mathrm{V}}\right)^{1 / 3}}$

which reveals a speed of sound for bulk Si of $5.71 \mathrm{~km} / \mathrm{s}$ for the literature Debye temperature of $625 \mathrm{~K}$ [33] and $6.81(2) \mathrm{km} / \mathrm{s}$ for the Debye temperature of $745(2) \mathrm{K}$. The calculated values for speed of sound for the nanocrystalline samples are summarized in Table 2.

\section{Elastic constants}

The values obtained for the elastic constants $C_{11}$ and $C_{44}$ with RUS are shown in Table 2 and the overall RMS errors between the calculated and measured frequencies were 0.784 and $0.586 \%$ (without excluding any resonance frequencies) for the samples with smaller and larger nanocrystallites, respectively. With those values, it was possible to extract the speed of sound using

$\frac{3}{v_{\mathrm{s}}^{3}}=\frac{1}{v_{\text {long }}^{3}}+\frac{2}{v_{\text {trans }}^{3}}$,

where $v_{\text {long }}=\sqrt{C_{11} / \rho}$ and $v_{\text {trans }}=\sqrt{C_{44} / \rho}$ for the polycrystals.

In contrast for single crystals, $v_{\text {long }}=\sqrt{G / \rho}$ and $v_{\text {trans }}=$

\begin{tabular}{|c|c|c|c|c|c|c|c|c|}
\hline & \multirow[t]{2}{*}{$e(\mathrm{~J} / \mathrm{g} \mathrm{K})$} & \multicolumn{2}{|l|}{$\Theta_{\mathrm{E}}$} & \multirow{2}{*}{$\begin{array}{l}b \\
(\mathrm{~J} / \mathrm{g} \mathrm{K})\end{array}$} & \multicolumn{2}{|l|}{$\Theta_{\mathrm{BP}}$} & \multirow[t]{2}{*}{$d(\mathrm{~J} / \mathrm{g} \mathrm{K})$} & \multirow[t]{2}{*}{$\Theta_{\mathrm{D}}(\mathrm{K})$} \\
\hline & & $(\mathrm{K})$ & $(\mathrm{meV})$ & & $(\mathrm{K})$ & $(\mathrm{meV})$ & & \\
\hline Bulk [30] & $0.20(1)$ & 202(3) & $17.4(3)$ & - & - & - & $0.69(1)$ & $745(2)$ \\
\hline$\varnothing 40 \mathrm{~nm}$ & $0.19(2)$ & 194(5) & 16.7(4) & $0.006(1)$ & $62(7)$ & $5.3(6)$ & $0.66(4)$ & $663(65)$ \\
\hline$\varnothing 30 \mathrm{~nm}$ & $0.18(2)$ & $197(5)$ & $16.9(4)$ & $0.009(1)$ & $63(8)$ & $5.5(7)$ & $0.65(5)$ & $636(64)$ \\
\hline
\end{tabular}
$\sqrt{\frac{B+\frac{4}{3} G}{\rho}}$. The average speed of sound is then obtained by

Table 1 Einstein temperatures and energies extracted from the heat capacity fit for the nanocrystalline Si samples and bulk reference 
Table 2 Summary of the elastic constants and sound velocities in crystalline, polycrystalline, and nanocrystalline Si obtained by different techniques

\begin{tabular}{|c|c|c|c|c|c|}
\hline \multirow{2}{*}{ ( } & \multirow{2}{*}{$C_{11}(\mathrm{GPa})$} & \multirow[t]{2}{*}{$C_{44}(\mathrm{GPa})$} & \multicolumn{3}{|c|}{$v_{\mathrm{s}}(\mathrm{km} / \mathrm{s})$} \\
\hline & & & RUS & DPS & $C_{\mathrm{p}}, \Theta_{D}$ \\
\hline Bulk & 160.1 [29] & 80.0 [29] & 5.94 & $6.73(5)$ & $6.81(2)$ \\
\hline $40 \mathrm{~nm}$ & $164(2)$ & $59.7(6)$ & $5.62(7)$ & $3.49^{\mathrm{a}}$ & $6.1(6)$ \\
\hline $30 \mathrm{~nm}$ & $147(2)$ & $52.7(5)$ & $5.42(6)$ & $3.30^{\mathrm{a}}$ & $5.8(6)$ \\
\hline
\end{tabular}

${ }^{a}$ The sound velocity of the nanocrystalline samples calculated from the DPS is only a lower limit value, as the presence of the Boson peak prevents proper evaluation of $g(E) / E^{2}$ for $E \rightarrow 0$ )

considering the bulk modulus $B=\frac{C_{11}+2 C_{12}}{3}=93.4 \mathrm{GPa}$, $C_{12}=57.8 \mathrm{GPa}$ for $\mathrm{Si}[29]$, and the shear modulus $G=66.9$ GPa calculated with the Hershey-KrönerEshelby averaging method. ${ }^{1}$

The calculated sound velocities are reported in Table 2.

$C_{11}$ is 164(2) and 147(2) GPa and $C_{44}$ is 59.7(6) and 52.7(5) $\mathrm{GPa}$ for the samples with 40 and $30 \mathrm{~nm}$ nanocrystallites, respectively. Compared to the values of single crystalline silicon [29], the $C_{11}$ is $3 \%$ larger for the sample with $40 \mathrm{~nm}$ nanocrystallites and $8 \%$ smaller for the sample with $30 \mathrm{~nm}$ nanocrystallites, whereas $C_{44}$ is 25 and $34 \%$ smaller for the samples with 40 and $30 \mathrm{~nm}$ nanocrystallites, respectively. Furthermore, a decrease of up to $16 \%$ of the speed of sound calculated from the elastic constants was obtained upon nanostructuration and the presence of impurities.

Shintani et al. [41] suggested a link between the Boson peak for glasses (observed in the reduced DPS and in the $C_{\mathrm{p}} / T^{3}$ ) and the Ioffe-Regel frequency limit for transverse phonons, above which transverse modes no longer propagate. Therefore, the presence of a significant amount of amorphous $\mathrm{SiO}_{2}$ on the sample and a substantial decrease of $C_{44}$ when compared to single crystal bulk $\mathrm{Si}$ [29] further reinforce the idea that the presence of an amorphous phase disturbs mainly the propagation of transverse, shear, waves.

\section{Low temperature transport properties}

Macroscopic measurements of the Seebeck coefficient and resistivity are shown in Fig. 6a and b, respectively, whereas thermal conductivity is shown in Fig. 7. An increase of the absolute values of the Seebeck coefficients with temperature is observed, whereas the resistivity is relatively constant with varying temperature as expected for a highly doped semiconductor, in agreement with the electronic contribution observed on the heat capacity at low temperatures.

\footnotetext{
${ }^{1}$ Hershey-Kröner-Eshelby described by Eshelby [40]: $G^{3}+\alpha$ $G^{2}+\beta \quad G+\gamma=0, \quad$ where $\quad \alpha=\frac{5 C_{11}+4 C_{12}}{8}, \beta=-\frac{C_{44}\left(7 C_{11}-4 C_{12}\right)}{8}$, $\gamma=-\frac{C_{44}\left(C_{11}-C_{12}\right)\left(C_{11}+2 C_{12}\right)}{8}$.
}

Room temperature values of the resistivity, Seebeck coefficient, and thermal conductivity for both pellets are summarized in Table 3, as well as the calculated values of power factor $\left(S^{2} \rho^{-1}\right)$ and dimensionless figure of merit (ZT), and are compared with previously reported values on nanostructured bulk Si by Bux et al. [8] and with heavily doped $n$-type polycrystalline $\mathrm{Si}$ [14].

The power factor $\left(S^{2} \rho^{-1}\right)$ at $295 \mathrm{~K}$ is larger for the sample with less oxides as the absolute value of the Seebeck coefficient is twice as large, and the resistivity is two-thirds of the value for the sample with more oxides content. When compared with heavily doped $n$-type polycrystalline $\mathrm{Si}$ [14], the sample with $40 \mathrm{~nm}$ nanocrystallites presents similar values of the Seebeck coefficient while the sample with $30 \mathrm{~nm}$ nanocrystallites presents similar values of the resistivity. The absolute value of the Seebeck coefficient of both samples were better than the values obtained for other nanostructured bulk Si prepared by a different approach [8], although the resistivity presented worse values.

Two effects can influence the value of the Seebeck coefficient in nanostructured materials: it can be reduced due to a a thermal boundary resistance between the grains leading to a temperature drop over the interfaces, or it can be enhanced due to electron-filtering effects at the interfaces [42]. Amorphous $\mathrm{SiO}_{2}$ present in small quantities can significantly enhance the Seebeck coefficient-either because the second interface effect is more prominent than the first or the phosphorus dopant could be incorporated preferentially within the oxide phase and is therefore partially lost for the Si.

Although the sample with more oxides (smaller nanocrystallites) presented a larger decrease in the thermal conductivity, the significantly larger power factor of the samples with less oxides (larger nanocrystallites) gives rise to an improved figure of merit at room temperature, which is comparable to the room temperature $\mathrm{ZT}$ previously obtained by Bux et al. [8].

Wang et al. [43] have previously measured the thermal conductivity of nanocrystalline $\mathrm{Si}$ with similar preparation methods and densities as the samples presented here but with a considerably lower content of oxidic precipitates. 
Fig. 6 Seebeck coefficient (a) and resistivity (b) for both nanocrystalline $\mathrm{Si}$ samples
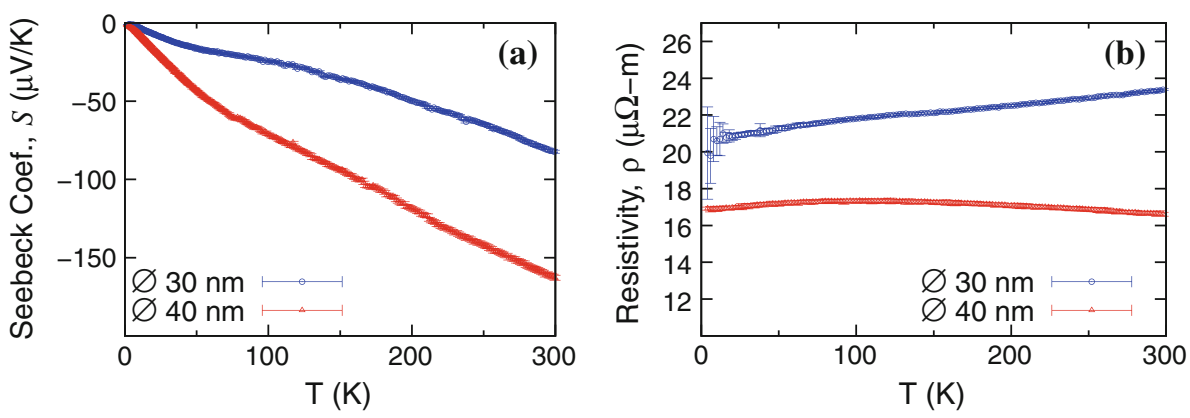

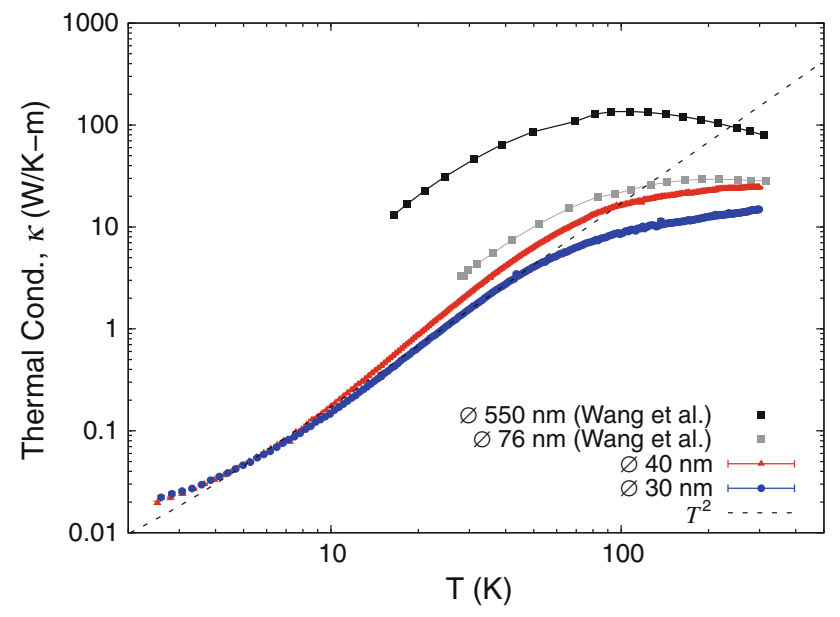

Fig. 7 Thermal conductivity for both nanocrystalline $\mathrm{Si}$ samples compared with Wang et al. [43]

Some of their results are compared with the values obtained in this study in Fig. 7. They observed a $T^{2}$ dependence at low temperatures which cannot be explained by a traditional model with frequency-independent grain boundary scattering, so called "gray", and proposed a new frequency-dependent model for the thermal conductivity suggesting that the mean free path for grain boundary scattering is inversely proportional to the phonon frequency. The data presented in this study features the same $T^{2}$ dependence as plotted in Fig. 7.

Furthermore, a calculation of the phononic contribution to the thermal conductivity $\left(\kappa_{\text {lat }}\right)$ was obtained by subtracting the electronic contribution $\left(\kappa_{\mathrm{el}}\right)$ from the total thermal conductivity, which can be obtained with the Wiedemann-Franz law:

$\kappa_{\mathrm{el}}=\frac{L T}{\rho}$,

where $L=2.44 \times 10^{-8} \mathrm{~W} \Omega \mathrm{K}^{-2}$ is the Lorenz number, $T$ is the temperature, and $\rho$ is the resistivity.

With the measured resistivity, the electronic contribution to the thermal conductivity was very low $\left(\kappa_{\mathrm{el}}=0.3-0.5\right.$ $\mathrm{W} / \mathrm{K} \mathrm{m}$ at $295 \mathrm{~K}$ ), and therefore the phononic contribution accounts for $98 \%$ of the total thermal conductivity of both samples at room temperature.

The thermal conductivity at room temperature is 156 W/K m for undoped single crystal Si [7] and decreases to $64.9 \mathrm{~W} / \mathrm{K} \mathrm{m}$ in heavily doped polycrystalline $\mathrm{Si}$ [44]. The nanocrystalline $\mathrm{Si}$ samples that we produced were also highly doped with phosphorus and present an even larger decrease of the thermal conductivity $(15 \mathrm{~W} / \mathrm{K} \mathrm{m})$, corresponding to $90 \%$ reduction when compared to single crystal Si and to $73 \%$ when compared to the doped sample. As expected, the sample which was produced with smaller nanoparticle size has a larger decrease on the thermal conductivity, as the surface area is larger and therefore it presents more oxide impurities.

On one hand, a large decrease of the thermal conductivity was observed upon nanostructuration not only due to grain boundaries but also due to the large amount of amorphous $\mathrm{SiO}_{2}$. On the other hand, such impurities also had an important impact on the thermopower factor $\left(S^{2} \rho^{-1}\right)$, resulting in a figure of merit much larger for the the sample with larger nanocrystallites $(0.02)$ than for the sample with higher impurities concentration (0.006).

Table 3 Room temperature thermoelectric properties of both nanostructured samples compared with nanostructured bulk Si previously reported by Bux et al. [8] and with heavily doped $n$-type polycrystalline Si [14]

\begin{tabular}{lllll}
\hline & $\varnothing 30 \mathrm{~nm}$ & $\varnothing 40 \mathrm{~nm}$ & Nanostructured [8] & Polycrystalline [14] \\
\hline Seebeck coeff. $(\mu \mathrm{V} / \mathrm{K})$ & $-81.2(9)$ & $-161(2)$ & -70 & -152 \\
Resistivity $(\mu \Omega \mathrm{m})$ & $23.7(3)$ & $16.5(9)$ & 9.1 & 27.3 \\
Thermal cond. $(\mathrm{W} / \mathrm{K} \mathrm{m})$ & $14.8(3)$ & $24.4(4)$ & 7.0 & 64.9 \\
Power factor $\left(\mu \mathrm{W} / \mathrm{K}^{2} \mathrm{~m}\right)$ & 0.2 & 1.6 & 0.5 & 0.8 \\
Figure of merit $(\mathrm{ZT})$ & $0.0055(2)$ & $0.019(1)$ & 0.023 & 0.004 \\
\hline
\end{tabular}


Further information on the high temperature thermoelectric properties of samples prepared with the same process as in this article can be found in [21].

Phonon mean free path $(\lambda)$

The phonon mean free path at room temperature could be calculated using the values obtained for the heat capacity $\left(C_{\mathrm{p}}\right)$, sound velocity extracted with RUS $\left(v_{\mathrm{s}}\right)$, and lattice thermal conductivity $\left(\kappa_{\text {lat }}\right)$ :

$\kappa_{\text {lat }}=\frac{N_{\mathrm{V}} v_{\mathrm{s}} \lambda C_{\mathrm{V}}}{3 N_{\mathrm{a}}}$

and it was found to be 8.3 and $5.4 \mathrm{~nm}$ for the samples with larger and smaller nanocrystallites, respectively.

Previous calculation of the phonon mean free path of $\mathrm{Si}$ was done as in this article and showed a strong dependence on the temperature and impurity concentration [45]. The phonon mean free path was larger in weakly P-doped $\mathrm{Si}$, decreasing with increasing temperature and reaching a value of $35 \mathrm{~nm}$ at room temperature, whereas in heavily As-doped $\mathrm{Si}$ it was temperature independent with a value around $15 \mathrm{~nm}$. The values for phonon mean free path for the heavily P-doped samples containing impurities are even lower than the ones reported for weakly P-doped Si and also for the heavily As-doped Si.

\section{Summary}

Two batches of phosphorus-doped silicon nanopowder with different nanoparticles sizes were produced by a gasphase synthesis and pressed into a pellet by spark plasma sintering after being exposed to air. Structural and chemical analyses of the samples by means of TEM, XRD, and PDF analyses shows a 9.6 and $15 \%$ concentration of impurities in the form of amorphous $\mathrm{SiO}_{2}$ agglomerated at the grain boundaries. The presence of additional peaks on the DPS obtained with inelastic neutron scattering when compared with a Si bulk reference suggests the presence of hydrogen in interstitial lattice sites in silicon and the reduced DPS showed a Boson peak at low energies due to the presence of amorphous $\mathrm{SiO}_{2}$ in the material, which was also observed on the heat capacity $\left(C_{\mathrm{p}}\right)$ divided by $T^{3}$. The $C_{\mathrm{p}}$ was modelled with a Debye term and two Einstein terms: one describing the excess $C_{\mathrm{p}}$ at $\sim 45 \mathrm{~K}$ due to a flat acoustic mode in the dispersion curve which is characteristic for any form of Si and the other describing the Boson contribution. The Einstein temperatures obtained with this fit are in very good agreement with the position of the same peak in the DPS. Measurement of the elastic constants with RUS showed a larger deviation of the constant $C_{44}$ when compared to bulk $\mathrm{Si}$, i.e., nanostructuration and the presence of impurities disturbs mainly the propagation of transverse (shear) waves, possibly related to the link between Boson peak in glasses and the propagation of transverse phonons previously suggested by Shintani et al. Measurements of the thermoelectric properties of the materials reveal a large decrease of the thermal conductivity which was more significant for the sample with smaller nanocrystallites (and larger amounts of impurities) but also a significant decrease on the power factor, resulting in a thermoelectric figure of merit enhanced by a factor of three compared to bulk silicon.

Acknowledgements The Institute Laue-Langevin (ILL-Grenoble, France) and the Advanced Photon Source (APS) at Argonne National Laboratory are acknowledged for neutron and synchrotron radiation beam time, respectively. The Helmholtz Gemeinschaft Deutscher Forschungszentren is acknowledged for funding VH NG-407 "Lattice dynamics in emerging functional materials." Z. Wang and J.E. Alaniz from UC Riverside are kindly acknowledged for sharing their data on thermal conductivity of nanocrystalline Si. D. Weber is acknowledged for scientific input and programming and S. Disch is acknowledged for helpful input for the PDF data analysis.

Open Access This article is distributed under the terms of the Creative Commons Attribution License which permits any use, distribution, and reproduction in any medium, provided the original author(s) and the source are credited.

\section{References}

1. Dresselhaus MS, Chen G, Tang MY, Yang RG, Lee H, Wang DZ, Ren ZF, Fleurial JP, Gogna P (2007) Adv Mater 19(8):1043. doi: 10.1002/adma.200600527. URL:http://dx.doi.org/10.1002/adma. 200600527

2. Wang XW, Lee H, Lan YC, Zhu GH, Joshi G, Wang DZ, Yang J, Muto AJ, Tang MY, Klatsky J, Song S, Dresselhaus MS, Chen G, Ren ZF (2008) Appl Phys Lett 93(19):193121. doi:10.1063/ 1.3027060. URL:http://apl.aip.org/applab/v93/i19/p193121_s1

3. Poudel B, Hao Q, Ma Y, Lan Y, Minnich A, Yu B, Yan X, Wang D, Muto A, Vashaee D, Chen X, Liu J, Dresselhaus MS, Chen G, Ren Z (2008) Science 320(5876):634. doi:10.1126/science.1156 446. URL:http://www.sciencemag.org/cgi/content/abstract/320/ $5876 / 634$

4. Zhu GH, Lee H, Lan YC, Wang XW, Joshi G, Wang DZ, Yang J, Vashaee D, Guilbert H, Pillitteri A, Dresselhaus MS, Chen G, Ren ZF (2009) Phys Rev Lett 102(19):196803. doi:10.1103/ PhysRevLett.102.196803

5. Hochbaum AI, Chen R, Delgado RD, Liang W, Garnett EC, Najarian M, Majumdar A, Yang P (2008) Nature 451(7175):163. doi:10.1038/nature06381. URL:http://dx.doi.org/10.1038/nature0 6381

6. Boukai AI, Bunimovich Y, Tahir-Kheli J, Yu JK, Goddard III WA, Heath JR (2008) Nature 451(7175):168. doi:10.1038/nature 06458. URL:http://dx.doi.org/10.1038/nature06458

7. Glassbrenner CJ, Slack GA (1964) Phys Rev 134(4A):A1058 doi: 10.1103/PhysRev.134.A1058. URL:http://link.aps.org/doi/10.1103/ PhysRev.134.A1058

8. Bux S, Blair R, Gogna P, Lee H, Chen G, Dresselhaus M, Kaner R, Fleurial JP (2009) Adv Funct Mater 19(15):2445. doi10.1002/ adfm. 200900250 
9. Lan Y, Minnich AJ, Chen G, Ren Z (2010) Adv Funct Mater 20:357

10. Gibbons T, Kang B, Estreicher S, Carbogno C (2011) Phys Rev B 84(3):035317. 10.1103/PhysRevB.84.035317

11. Thompson J, Younglove B (1961) J Phys Chem Solids 20(12): 146. URL:http://www.sciencedirect.com/science/article/pii/0022 369761901469

12. Schierning G, Theissmann R, Stein N, Petermann N, Becker A, Engenhorst M, Kessler V, Geller M, Beckel A, Wiggers H, Schmechel R (2011) J Appl Phys 110(11):113515. 10.1063/1. 3658021

13. Knipping J, Wiggers H, Rellinghaus B, Roth P, Konjhodzic D, Meier C (2004) J Nanosci Nanotechnol 4(8):1039. 10.1166/ jnn.2004.149

14. Stegner A, Pereira R, Klein K, Wiggers H, Brandt M, Stutzmann M (2007) Physica B 401-402:541. doi:10.1016/j.physb.2007.09. 017. URL:http://www.sciencedirect.com/science/article/B6TVH4PMJK2K-1/2/d98384c5cf87ee6826f919d74688ad62.

15. Rodriguez-Carvajal J (2009) Fullprof, Tech. report, Laboratoire Leon Brillouin (CEA-CNRS), France

16. Hammersley AP (1997) Fit2d: an introduction and overview. ESRF Internal Report ESRF97HA02T, ESRF

17. Qiu X, Thompson JW, Billinge SJL (2004) J Appl Crystallogr 37(4):678 doi:10.1107/S0021889804011744. URL:http://dx.doi. org/10.1107/S0021889804011744

18. Farrow CL, Juhas P, Liu JW, Bryndin D, Boin ES, Bloch J, Proffen T, Billinge SJL (2007) J Phys 19(33):335219. URL: http://stacks.iop.org/0953-8984/19/i=33/a=335219

19. LAMP, the Large Array Manipulation Program. URL:http:// www.ill.fr/data_treat/lamp/lamp.html

20. Migliori A, Sarrao JL (1997) Resonant ultrasound spectroscopy: applications to physics, materials measurements, and nondestructive evaluation, 1st edn. Wiley-Interscience, New York

21. Schierning G, Claudio T, Theissmann R, Stein N, Petermann N, Becker A, Denker J, Wiggers H, Hermann R, Schmechel R. In: MRS Proceedings, vol 1267, p DD01. doi:10.1557/PROC1267-DD01-09. URL:http://journals.cambridge.org/action/display Abstract? fromPage $=$ online $\&$ aid $=7956787$

22. Chumakov AI, Monaco G, Monaco A, Crichton WA, Bosak A, Rüffer R, Meyer A, Kargl F, Comez L, Fioretto D, Giefers H, Roitsch S, Wortmann G, Manghnani MH, Hushur A, Williams Q, Balogh J, Parliński K, Jochym P, Piekarz P (2011) Phys Rev Lett 106(22): 225501. URL:http://link.aps.org/doi/10.1103/PhysRevLett.106. 225501

23. Malinovsky V, Novikov V, Surovtsev N, Shebanin A (2000) Phys Solid State 42:65. URL:http://dx.doi.org/10.1134/1.1131169. 10.1134/1.1131169

24. Fabiani E, Fontana A, Buchenau U (2008) J Chem Phys 128(24):244507. doi:10.1063/1.2937731

25. Wright AC, Hannon AC, Sinclair RN, Brunier TM, Guy CA, Stewart RJ, Strobel MB, Jansen F (2007) J Phys 19(41):415109. URL:http://stacks.iop.org/0953-8984/19/i=41/a=415109

26. Kamitakahara WA, Shanks HR, McClelland JF, Buchenau U, Gompf F, Pintschovius L (1984) Phys Rev Lett 52(8):644. URL: http://link.aps.org/doi/10.1103/PhysRevLett.52.644
27. Stavola M (2008) In: The 5th international symposium on advanced science and technology of silicon materials (JSPS Si Symposium). URL:http://fukuoka.riam.kyushu-u.ac.jp/hawaii 2008/pdf/O-01.pdf

28. Chumakov A, Bosak A, Ruffer R (2009) Phys Rev B 80(9): 094303. doi:10.1103/PhysRevB.80.094303

29. Madelung O, Rössler U, Schulz M (eds.) (2011) Landolt-Börnstein-Group III condensed matter. In: Silicon (Si) elastic moduli of Si-I, vol. 41A1a. SpringerMaterials-The Landolt-Börnstein Database. doi:10.1007/10551045_212

30. Flubacher P, Leadbetter AJ, Morrison JA (1959) Philos Mag 4(39):273. doi:10.1080/14786435908233340. URL:http://www. informaworld.com/10.1080/14786435908233340

31. Flubacher P, Leadbetter A, Morrison J, Stoicheff B (1959) J Phys Chem Solids 12(1):53. URL:http://www.sciencedirect.com/science/ article/pii/0022369759902513

32. Touloukian YS, Buyco EH (1970) Thermophysical properties of matter, vol 5. IFI/Plenum, New York

33. Ashcroft NW, Mermin ND (1976) Solid state physics. Thomson Learning, Inc., London

34. Lubchenko V, Wolynes PG (2003) Proc Natl Acad Sci USA 100(4):1515. doi:10.1073/pnas.252786999

35. Kittel C (1995) Introduction to solid state physics, 7th edn. Wiley, New York

36. Wang L, Tan Z, Meng S, Druzhinina A, Varushchenko RA, Li G (2001) J Non-Cryst Solids 296(12):139. doi:10.1016/S0022-3093 (01)00902-4. URL:http://www.sciencedirect.com/science/article/pii/ S0022309301009024

37. Mertig M, Pompe G, Hegenbarth E (1984) Solid State Commun 49(4):369. URL:http://www.sciencedirect.com/science/article/pii/ 0038109884905891

38. Zdetsis AD, Wang CS (1979) Phys Rev B 19(6):2999. URL: http://link.aps.org/doi/10.1103/PhysRevB.19.2999

39. Hermann RP, Grandjean F, Long GJ (2005) Am J Phys 73(2):110. URL:http://dx.doi.org/10.1119/1.1783899

40. Eshelby JD (1961) Progress in solid mechanics, vol 2. NorthHolland Publishing Company, Amsterdam. p 130

41. Shintani H, Tanaka H (2008) Nat Mater 7(11):870. URL:http:// dx.doi.org/10.1038/nmat2293

42. Zide JMO, Vashaee D, Bian ZX, Zeng G, Bowers JE, Shakouri A, Gossard AC (2006) Phys Rev B 74:205335. URL:http://link. aps.org/doi/10.1103/PhysRevB.74.205335.10.1103/PhysRevB.74. 205335

43. Wang Z, Alaniz JE, Jang W, Garay JE, Dames C (2011) Nano Lett 11(6):2206. URL:http://dx.doi.org/10.1021/nl1045395

44. Gaidry THT (1967) Thermal conductivity, seebeck coefficient and electrical resistivity of heavily phosphorus-doped silicon from 313k to 673k. Technical Report 23, South Dakota School of Mines and Technology

45. Weber L, Gmelin E (1991) Appl Phys A 53:136. URL:http://dx. doi.org/10.1007/BF00323873.10.1007/BF00323873 